\title{
Avaliação de serviços de saúde bucal: um estudo comparativo
}

Evaluation of oral health services: a comparative study

Aline Lütkemeyer Brunhauser

Miriam Lago Magro**

Matheus Neves ${ }^{* * *}$

\section{Resumo}

Introdução: a avaliação em saúde no Brasil não é tida como rotina, porém, é ela quem fornece subsídios e confere suporte ao planejamento e à tomada de decisões do Sistema de Saúde. Objetivo: avaliar o acesso e a satisfação dos usuários dos serviços odontológicos do SUS, comparando usuários da Estratégia de Saúde da Família (ESF) com usuários de UBSs convencionais, em Não-Me-Toque/RS, Brasil. Métodos: tratou-se de um estudo transversal de base populacional, com amostra de 280 indivíduos, identificados em seus domicílios por meio de amostragem por conglomerado e usuários dos serviços públicos de Saúde Bucal. Utilizou-se o Questionnaire for Quality Evaluation of Oral Health (QASSaB) - Questionário de Avaliação da Qualidade dos Serviços de Saúde Bucal, devidamente validado para os serviços brasileiros. Resultados: testes de qui-quadrado demonstraram que 93\% dos usuários da ESF apresentaram-se muito/totalmente satisfeitos e apenas $7 \%$ mostraram-se satisfeitos. Já no grupo de usuários das UBSs convencionais, $86 \%$ apresentaram-se muito/ totalmente satisfeitos e outros $14 \%$ mostraram-se satisfeitos. Conclusão: em Não-Me-Toque/RS, os usuários cobertos pela ESF apresentam uma satisfação maior com os serviços de saúde bucal que os usuários cobertos pelas UBSs convencionais.

Palavras-chave: Satisfação do paciente. Atenção primária à saúde. Avaliação em saúde. Serviços de saúde.

\section{Introdução}

Durante muitos anos, no Brasil, a inserção da saúde bucal e das práticas odontológicas no Sistema Único de Saúde (SUS) deu-se de forma paralela e afastada do processo de organização dos demais serviços de saúde. Atualmente, essa tendência vem sendo revertida, observando-se o esforço para promover uma maior integração da saúde bucal nos serviços de saúde em geral, a partir da conjugação de saberes e práticas que apontem para a promoção e vigilância em saúde, para revisão das práticas assistenciais que incorporam a abordagem familiar e a defesa da vida ${ }^{1}$.

Com o surgimento da Saúde da Família como modelo de atenção reogarnizador da Atenção Primária à Saúde, verifica-se uma nova forma de atuar, com propostas para mudar toda a antiga concepção de atuação dos profissionais de saúde, passando a atuar na integralidade da assistência, de modo a tratar o indivíduo como sujeito dentro da sua realidade socioeconômica e cultural, considerando essa dimensão globalizante ${ }^{2}$.

É relevante, pois, investigar quais são as ideias, os valores e as crenças desenvolvidas pelos usuários acerca do atual quadro de atenção à saúde, entendendo o usuário como um interlocutor válido e como agente social das próprias mudanças por meio de reflexão consciente e compartilhada do problema ${ }^{3}$. Além disso, a avaliação em saúde tem como propósito fundamental dar suporte a todo o processo decisório no âmbito do Sistema de Saúde e, por isso, deve subsidiar a identificação de problemas e a reorientação de ações e serviços desenvolvidos, avaliar a incorporação de novas práticas sanitárias na rotina de profissionais e mensurar o impacto das ações 
implementadas pelos serviços e programas sobre 0 estado de saúde da população $0^{1,4}$.

Portanto, o presente estudo tem como objetivo investigar se os serviços odontológicos incorporados à Estratégia de Saúde da Família (ESF) geram maior satisfação aos seus usuários, quando em comparação aos serviços odontológicos não incorporados à ESF, na cidade de Não-Me-Toque/RS, Brasil.

\section{Métodos}

Tratou-se de um estudo transversal de base populacional, com amostra representativa de homens e mulheres, identificados em seus domicílios por meio de amostragem por conglomerado e moradores do município de Não-Me-Toque/RS.

A cidade de Não-Me-Toque está situada na região do Alto Jacuí, no estado do Rio Grande do Sul, região Sul do Brasil, com uma população estimada pelo IBGE (2010) em 15.936 habitantes. Sua divisão territorial dá-se em 17 bairros, dos quais, 7 possuem Unidades Básicas de Saúde (UBSs), destes, 2 com ESF.

A amostra foi estimada num total de 276 pessoas, sendo 113 moradores de bairros com cobertura da ESF e os outros 163, moradores de bairros sem cobertura da ESF e que utilizam UBS. A amostra foi ampliada em $10 \%$, considerando eventuais perdas.

Para estimar o tamanho amostral, foram utilizadas as prevalências e os resultados encontrados no estudo "Avaliação da satisfação dos usuários dos serviços odontológicos do SUS, Curitiba, Paraná, Brasil" . Além disso, foi considerado um intervalo de confiança de $95 \%$, admitindo-se um erro $\beta$ de $20 \%$ e consecutivo poder estatístico de $80 \%$. Também, levou-se em consideração a porcentagem proporcional da cobertura de serviços públicos de saúde do município.

Os critérios de inclusão para participação no estudo foram que o participante deveria ser morador daquele domicílio sorteado, não ser morador temporário, utilizar o serviço odontológico público e aceitar participar do estudo. Nos casos de domicílios em que havia mais de um morador elegível, todos foram convidados a participar.

Realizou-se uma amostragem aleatória proporcional por conglomerado, seguindo os setores censitários, que são subdivisões geográficas do município estabelecidas pelo Instituto Brasileiro de Geografia e Estatística (IBGE). O setor censitário serviu como referência para compor a amostra total de maneira proporcional à porcentagem de cobertura da ESF e da UBS. A definição do quarteirão elencado como ponto de partida para as entrevistas, em cada setor, foi baseada em um fluxograma elaborado com base nos mapas dos setores. Os quarteirões foram numerados, e definiu-se a sequência dos quarteirões percorridos. Em cada quarteirão, foi sorteada, aleatoriamente, uma esquina que representou o ponto inicial. A partir desse ponto, iniciaram-se as visitas pela primeira casa e, a seguir, sempre no sentido da esquerda de quem está de frente para a esquina inicial, saltou-se uma casa, incluindo-se o próximo domicílio, até obter todas as entrevistas possíveis naquele setor censitário. Nos casos em que o número de entrevistas do setor não foi alcançado no respectivo quarteirão, prosseguiu-se para o próximo quarteirão, na ordem sorteada, até a obtenção de todas as entrevistas.

O instrumento de pesquisa utilizado foi o Questionnaire for Quality Evaluation of Oral Health (QASSaB) - Questionário de Avaliação da Qualidade dos Serviços de Saúde Bucal, o qual foi construído para os serviços brasileiros, estando devidamente validado pelo estudo "Validação de um instrumento para avaliação da satisfação dos usuários, com os serviços públicos de saúde bucal - QASSaB"6. Tal questionário contempla um modelo de avaliação distribuído em sete dimensões: relações humanas, eficácia, acessibilidade, qualidade técnico-científica, ambiente físico/limpeza, aceitabilidade e resolutividade.

Para aferir essas sete dimensões psicométricas, o QASSaB utiliza 11 questões (Q), a saber: (1) relações humanas, referentes ao tratamento dispensado pelo dentista (Q1) e por outros profissionais (auxiliar do dentista - Q2); (2) eficácia, relacionada ao incômodo após o tratamento (Q3); (3) acessibilidade, apurada pela obtenção de uma vaga (Q4) e pelo tempo de espera no dia do atendimento (Q5); (4) qualidade técnico-científica, em relação à atualização tecnológica dos equipamentos odontológicos (Q6); (5) ambiente físico, ou seja, limpeza da recepção (Q7) e de banheiros (Q8); (6) aceitabilidade, referente à explicação ao usuário sobre o tratamento mais adequado (Q9); (7) resolutividade, relativa à satisfação com a aparência $(\mathrm{Q} 10)$ e capacidade de mastigação dos dentes tratados (Q11). As possibilidades de resposta ao instrumento foram construídas com base na escala de Likert ${ }^{6}$.

Além disso, foi aplicado um questionário sociodemográfico, para identificação da realidade social e econômica do participante. Por meio desse instrumento, os participantes foram questionados quanto à data de nascimento, sexo, raça, estado marital, anos de estudo, renda e suficiência da renda.

As variáveis contínuas e discretas foram descritas por meio da média e do respectivo desvio padrão, enquanto as variáveis categóricas foram descritas na forma de porcentagens absoluta e relativa. A existência de diferenças estatisticamente significativas em relação aos desfechos estudados foi verificada por meio do teste qui-quadrado.

De posse das respostas referentes ao QASSaB, foi construído um escore médio de satisfação por indivíduo participante do estudo. Assim, foi obtida a média aritmética das respostas dadas às perguntas constantes no instrumento de coleta de dados, os quais representaram a satisfação dos usuários com 
os serviços de saúde acessados. Essas médias foram classificadas como: Insatisfeito/Pouco Satisfeito (de 0 a 2,0); Satisfeito (de 2,0001 a 3,0); Muito Satisfeito/Totalmente Satisfeito (de 3,0001 a 5,0). Com base em tais dados, foi feita a avaliação da média das dimensões de qualidade.

O projeto teve aprovação do Comitê de Ética em Pesquisa da Universidade de Passo Fundo, conforme parecer $n^{\circ} 088 / 2011$, sendo igualmente aprovado pelo Conselho Municipal de Saúde do Município de Não-Me-Toque.

Os potenciais participantes desse inquérito foram convidados e receberam esclarecimentos quanto aos objetivos do estudo. Aqueles que cumpriram os critérios de inclusão e aceitaram participar foram convidados a ler e assinar o termo de consenti- mento livre e esclarecido (TCLE) e, ao final, responderam ao questionário padronizado deste estudo. A coleta de dados foi realizada pela pesquisadora, por meio de entrevista estruturada, entre maio e julho de 2011.

\section{Resultados}

A primeira tabela apresentada refere-se aos dados de caracterização da amostra estudada (Tabela 1). Reitera-se que a renda familiar esteve associada ao desfecho de satisfação com o serviço público de saúde bucal. A renda familiar declarada pelos participantes variou de $\mathrm{R} \$ 0,00$ a $\mathrm{R} \$ 5.000,00$, sendo a renda média $\mathrm{R} \$ 1.413,00$, com desvio padrão de \pm $\mathrm{R} \$ 737,20$ e mediana de $\mathrm{R} \$ 1.200,00$.

Tabela 1 - Caracterização da amostra de 280 usuários (113 ESF, 163 UBS) atendidos no município de Não-Me-Toque/RS, Brasil, 2011

\begin{tabular}{|c|c|c|c|c|c|c|c|}
\hline \multirow{2}{*}{ Variável independente } & \multicolumn{2}{|c|}{ ESF } & \multicolumn{2}{|c|}{ UBS } & \multirow[t]{2}{*}{ Valor de $\mathrm{p}^{*}$} & \multicolumn{2}{|c|}{ Grupo total } \\
\hline & $N$ & $\%$ & $\mathrm{~N}$ & $\%$ & & $N$ & $\%$ \\
\hline \multicolumn{8}{|l|}{ Faixa etária $(\text { anos) })^{* *}$} \\
\hline $15-30$ & 30 & $26 \%$ & 43 & $26 \%$ & 0,79 & 73 & $26 \%$ \\
\hline $31-50$ & 44 & $38 \%$ & 67 & $41 \%$ & & 111 & $40 \%$ \\
\hline $51-88$ & 42 & $36 \%$ & 53 & $33 \%$ & & 95 & $34 \%$ \\
\hline \multicolumn{8}{|l|}{ Gênero } \\
\hline Masculino & 30 & $26 \%$ & 31 & $19 \%$ & 0,18 & 61 & $22 \%$ \\
\hline Feminino & 87 & $74 \%$ & 132 & $81 \%$ & & 219 & $78 \%$ \\
\hline \multicolumn{8}{|l|}{ Raça } \\
\hline Branca & 102 & $88 \%$ & 154 & $94 \%$ & 0,07 & 256 & $91 \%$ \\
\hline Preta & 14 & $12 \%$ & 9 & $6 \%$ & & 23 & $8 \%$ \\
\hline Parda & 1 & $1 \%$ & 0 & $0 \%$ & & 1 & $0 \%$ \\
\hline \multicolumn{8}{|l|}{ Estado marital } \\
\hline Solteiro & 12 & $10 \%$ & 24 & $15 \%$ & 0,37 & 36 & $13 \%$ \\
\hline Casado/Morando junto & 80 & $68 \%$ & 112 & $69 \%$ & & 192 & $69 \%$ \\
\hline Separado/Divorciado & 11 & $9 \%$ & 16 & $10 \%$ & & 27 & $10 \%$ \\
\hline Viúvo & 14 & $12 \%$ & 11 & $7 \%$ & & 25 & $9 \%$ \\
\hline \multicolumn{8}{|l|}{ Escolaridade } \\
\hline Analfabeto & 5 & $4 \%$ & 3 & $2 \%$ & 0,09 & 8 & $3 \%$ \\
\hline Baixo nível de escolaridade & 81 & $69 \%$ & 96 & $59 \%$ & & 177 & $63 \%$ \\
\hline Médio nível de escolaridade & 29 & $25 \%$ & 57 & $35 \%$ & & 86 & $31 \%$ \\
\hline Alto nível de escolaridade & 2 & $2 \%$ & 7 & $4 \%$ & & 9 & $3 \%$ \\
\hline \multicolumn{8}{|l|}{ Renda familiar } \\
\hline Até um salário mínimo & 7 & $6 \%$ & 2 & $1 \%$ & 0,02 & 9 & $3 \%$ \\
\hline De um a dois salários mínimos & 47 & $40 \%$ & 49 & $30 \%$ & & 96 & $34 \%$ \\
\hline De dois a três salários mínimos & 30 & $26 \%$ & 53 & $33 \%$ & & 83 & $30 \%$ \\
\hline Mais de três salários mínimos & 33 & $28 \%$ & 59 & $36 \%$ & & 92 & $33 \%$ \\
\hline \multicolumn{8}{|l|}{ Suficiência da renda } \\
\hline Sim & 43 & $37 \%$ & 67 & $41 \%$ & 0,46 & 110 & $39 \%$ \\
\hline Não & 74 & $63 \%$ & 96 & $59 \%$ & & 170 & $61 \%$ \\
\hline
\end{tabular}

"Teste qui-quadrado com $5 \%$ de significância.

"Para um indivíduo, não foi possível coletar o dado idade. 
Na Tabela 2, estão descritos os resultados referentes à satisfação dos usuários dos serviços de saúde bucal do município, segundo todas as dimensões integrantes do QASSaB: relações humanas, eficácia, acessibilidade, qualidade técnico-científica, limpeza, aceitabilidade e resolutividade. Nesse momento, ainda não são feitas distinções entre as modalidades de serviço odontológico acessado; apenas tem-se o panorama geral da avaliação de qualidade referida pelos usuários do município.

Tabela 2 - Avaliação geral da satisfação dos usuários dos serviços de saúde bucal do SUS de Não-Me-Toque/RS, Brasil, 2011

\begin{tabular}{|c|c|c|c|c|c|c|}
\hline \multicolumn{6}{|l|}{ Dimensão de qualidade } & \multirow[b]{2}{*}{$\begin{array}{l}\text { Total } \\
\mathrm{n}(\%)\end{array}$} \\
\hline $\begin{array}{l}\text { Dimensão } 1 \text { - Relações } \\
\text { humanas }\end{array}$ & $\begin{array}{l}\text { Excelente (5) } \\
\text { n (\%) }\end{array}$ & $\begin{array}{c}\text { Boa }(4) \\
\text { n }(\%)\end{array}$ & $\begin{array}{l}\text { Regular (3) } \\
\text { n (\%) }\end{array}$ & $\begin{array}{c}\text { Ruim (2) } \\
\text { n (\%) }\end{array}$ & $\begin{array}{c}\text { Péssima (1) } \\
\mathrm{n}(\%)\end{array}$ & \\
\hline $\begin{array}{c}\text { Atenção recebida } \\
\text { pelo cirurgião-dentista }\end{array}$ & $71(25,4)$ & $167(59,6)$ & $34(12,1)$ & $6(2,1)$ & $2(0,7)$ & $280(100)$ \\
\hline \multirow{2}{*}{ Dimensão 2 - Eficácia } & $\begin{array}{l}\text { Não senti } \\
\text { nada (5) }\end{array}$ & $\begin{array}{c}\text { Pouco } \\
\text { desconforto (4) }\end{array}$ & $\begin{array}{c}\text { Senti } \\
\text { desconforto (3) }\end{array}$ & $\begin{array}{c}\text { Muito } \\
\text { desconforto (2) }\end{array}$ & $\begin{array}{c}\text { Total } \\
\text { desconforto (1) }\end{array}$ & Total \\
\hline & n $(\%)$ & $\mathrm{n}(\%)$ & n $(\%)$ & $\mathrm{n}(\%)$ & n $(\%)$ & n $(\%)$ \\
\hline Desconforto & $257(91,8)$ & $0(0)$ & $4(1,4)$ & $14(5)$ & $5(1,8)$ & $280(100)$ \\
\hline \multirow[t]{2}{*}{$\begin{array}{l}\text { Dimensão } 3 \text { - Acesso/ } \\
\text { Acessibilidade }\end{array}$} & Muito fácil (5) & Fácil (4) & $\begin{array}{c}\text { Nem fácil, } \\
\text { nem difícil (3) }\end{array}$ & Difícil (2) & Muito difícil (1) & Total \\
\hline & $\mathrm{n}(\%)$ & n $(\%)$ & n $(\%)$ & n $(\%)$ & n $(\%)$ & n (\%) \\
\hline Obtenção de vaga & $4(1,4)$ & $98(35)$ & $63(22,5)$ & $95(33,9)$ & $20(7,1)$ & $280(100)$ \\
\hline \multirow[t]{2}{*}{$\begin{array}{l}\text { Dimensão } 3 \text { - } \\
\text { Acesso/ Acessibilidade }\end{array}$} & Muito curto (5) & Curto (4) & $\begin{array}{c}\text { Nem longo, } \\
\text { nem curto (3) }\end{array}$ & Longo (2) & Muito longo (1) & Total \\
\hline & n (\%) & n (\%) & $\mathrm{n}(\%)$ & n (\%) & $\mathrm{n}(\%)$ & $\mathrm{n}(\%)$ \\
\hline Tempo de espera & $7(2,5)$ & $91(32,5)$ & $75(26,8)$ & $88(31,4)$ & $19(6,8)$ & $280(100)$ \\
\hline \multirow{2}{*}{$\begin{array}{l}\text { Dimensão } 4 \text { - Qualidade } \\
\text { técnico- } \\
\text { científica }\end{array}$} & $\begin{array}{c}\text { Supermodernos } \\
(5)\end{array}$ & Modernos (4) & $\begin{array}{c}\text { Nem uma } \\
\text { coisa, } \\
\text { nem outra (3) }\end{array}$ & $\begin{array}{l}\text { Ultrapassados } \\
\text { (2) }\end{array}$ & $\begin{array}{l}\text { Desatualizados } \\
\text { (1) }\end{array}$ & Total \\
\hline & $\mathrm{n}(\%)$ & $\mathrm{n}(\%)$ & $\mathrm{n}(\%)$ & $\mathrm{n}(\%)$ & $\mathrm{n}(\%)$ & n (\%) \\
\hline Equipamento & $5(1,8)$ & $172(61,4)$ & $77(27,5)$ & $18(6,4)$ & $8(2,9)$ & $280(100)$ \\
\hline Dimensão 5- Limpeza & $\begin{array}{c}\text { Excelente (5) } \\
\text { n (\%) }\end{array}$ & $\begin{array}{c}\text { Boa (4) } \\
\text { n (\%) }\end{array}$ & $\begin{array}{c}\text { Regular (3) } \\
\text { n (\%) }\end{array}$ & $\begin{array}{c}\text { Ruim (2) } \\
\text { n (\%) }\end{array}$ & $\begin{array}{c}\text { Péssima (1) } \\
\text { n (\%) }\end{array}$ & $\begin{array}{l}\text { Total } \\
\text { n (\%) }\end{array}$ \\
\hline Recepção & $73(26,1)$ & $167(59,6)$ & $38(13,6)$ & $2(0,7)$ & $0(0)$ & $280(100)$ \\
\hline Banheiros & $70(25)$ & $165(58,9)$ & $38(13,6)$ & $4(1,4)$ & $3(1,1)$ & $280(100)$ \\
\hline \multirow{2}{*}{$\begin{array}{l}\text { Dimensão 6- } \\
\text { Aceitabilidade } \\
\text { Explicação do } \\
\text { tratamento }\end{array}$} & $\begin{array}{c}\text { Sempre } \\
\text { me explica (5) }\end{array}$ & $\begin{array}{l}\text { Maioria das } \\
\text { vezes me } \\
\text { explica(4) }\end{array}$ & $\begin{array}{l}\text { Não me } \\
\text { lembro (3) }\end{array}$ & $\begin{array}{l}\text { Raras vezes } \\
\text { me explica (2) }\end{array}$ & $\begin{array}{l}\text { Nunca me } \\
\text { explica (1) }\end{array}$ & Total \\
\hline & $\begin{array}{c}\mathrm{n}(\%) \\
226(80,7)\end{array}$ & $\begin{array}{l}\text { n (\%) } \\
14(6)\end{array}$ & $\begin{array}{l}\mathrm{n}(\%) \\
2(0,7)\end{array}$ & $\begin{array}{c}\mathrm{n}(\%) \\
29(10,4)\end{array}$ & $\begin{array}{l}\mathrm{n}(\%) \\
9(3,2)\end{array}$ & $\begin{array}{c}\mathrm{n}(\%) \\
280(100)\end{array}$ \\
\hline \multirow{3}{*}{$\begin{array}{l}\text { Dimensão } 7 \text { - } \\
\text { Efetividade/ } \\
\text { Resolutividade } \\
\text { Aparência dos dentes }\end{array}$} & $\begin{array}{l}\text { Totalmente } \\
\text { satisfeito (5) }\end{array}$ & $\begin{array}{c}\text { Muito } \\
\text { satisfeito (4) }\end{array}$ & Satisfeito (3) & $\begin{array}{l}\text { Um pouco } \\
\text { satisfeito (2) }\end{array}$ & Insatisfeito (1) & Total \\
\hline & n (\%) & $\mathrm{n}(\%)$ & n (\%) & n (\%) & $\mathrm{n}(\%)$ & n $(\%)$ \\
\hline & $11(3,9)$ & $32(11,9)$ & $154(55)$ & $37(13,2)$ & $46(16,4)$ & $280(100)$ \\
\hline $\begin{array}{c}\text { Capacidade de } \\
\text { mastigação }\end{array}$ & $16(5,7)$ & $37(13,2)$ & $154(55)$ & $35(12,5)$ & $38(13,6)$ & $280(100)$ \\
\hline
\end{tabular}

Ao realizarem-se as comparações entre as diferentes modalidades de serviço de saúde bucal acessado, equipe de saúde bucal na ESF ou serviço de saúde bucal em UBS convencional, foi possível notar algumas diferenças entre a satisfação referida pelos respectivos usuários. A Tabela 3 apresenta as dimensões de qualidade que apresentaram diferença estatisticamente significativa nessas comparações. 
Tabela 3 - Avaliação comparativa da satisfação dos usuários dos serviços odontológicos do SUS entre Estratégia Saúde da Família (ESF) e Unidade Básica de Saúde (UBS) na cidade de Não-Me-Toque/RS, Brasil, 2011

\begin{tabular}{|c|c|c|c|c|c|}
\hline \multirow{2}{*}{ Dimensão 1 - Relações humanas } & \multicolumn{2}{|c|}{ ESF } & \multicolumn{2}{|c|}{ UBS } & \multirow[t]{2}{*}{ Valor de $\mathrm{p}^{*}$} \\
\hline & $\mathrm{n}$ & $\%$ & $\mathrm{n}$ & $\%$ & \\
\hline \multicolumn{6}{|l|}{ Atenção recebida pelos outros profissionais } \\
\hline Excelente & 44 & $38 \%$ & 24 & $15 \%$ & $<0,001$ \\
\hline Boa & 59 & $50 \%$ & 89 & $55 \%$ & \\
\hline Regular & 11 & $9 \%$ & 37 & $23 \%$ & \\
\hline Ruim/ Péssima & 3 & $3 \%$ & 13 & $8 \%$ & \\
\hline Total & 117 & $100 \%$ & 163 & $100 \%$ & \\
\hline \multicolumn{6}{|l|}{ Dimensão 3 - Acesso } \\
\hline \multicolumn{6}{|l|}{ Obtenção de vaga } \\
\hline Fácil/ Muito fácil & 53 & $45 \%$ & 49 & $30 \%$ & 0,03 \\
\hline Nem difícil, nem fácil & 22 & $19 \%$ & 41 & $25 \%$ & \\
\hline Difícil/ Muito difícil & 42 & $36 \%$ & 73 & $45 \%$ & \\
\hline Total & 117 & $100 \%$ & 163 & $100 \%$ & \\
\hline \multicolumn{6}{|l|}{ Dimensão 5 - Ambiente físico/ Limpeza } \\
\hline \multicolumn{6}{|l|}{ Recepção-Sala de espera } \\
\hline Excelente & 40 & $34 \%$ & 33 & $20 \%$ & 0,04 \\
\hline Boa & 63 & $54 \%$ & 104 & $64 \%$ & \\
\hline Regular & 14 & $12 \%$ & 24 & $15 \%$ & \\
\hline Ruim/ Péssima & 0 & $0 \%$ & 2 & $1 \%$ & \\
\hline Total & 117 & $100 \%$ & 163 & $100 \%$ & \\
\hline \multicolumn{6}{|l|}{ Banheiros } \\
\hline Excelente & 40 & $34 \%$ & 30 & $18 \%$ & 0,01 \\
\hline Boa & 61 & $52 \%$ & 104 & $64 \%$ & \\
\hline Regular & 15 & $13 \%$ & 23 & $14 \%$ & \\
\hline Ruim/ Péssima & 1 & $1 \%$ & 6 & $4 \%$ & \\
\hline Total & 117 & $100 \%$ & 163 & $100 \%$ & \\
\hline \multicolumn{6}{|l|}{ Dimensão 7 - Resolutividade } \\
\hline \multicolumn{6}{|l|}{ Capacidade de mastigação } \\
\hline Totalmente satisfeito & 11 & $9 \%$ & 5 & $3 \%$ & 0,001 \\
\hline Muito satisfeito & 8 & $7 \%$ & 29 & $18 \%$ & \\
\hline Satisfeito & 58 & $50 \%$ & 96 & $59 \%$ & \\
\hline Um pouco satisfeito & 17 & $15 \%$ & 18 & $11 \%$ & \\
\hline Insatisfeito & 23 & $20 \%$ & 15 & $9 \%$ & \\
\hline Total & 117 & $100 \%$ & 163 & $100 \%$ & \\
\hline
\end{tabular}

*Teste qui-quadrado com $5 \%$ de significância

No que se refere à primeira dimensão, relações humanas, a maioria dos usuários da ESF (88\%) referiu a atenção recebida pelo outros profissionais como excelente ou boa, enquanto na UBS convencional esse percentual foi de $70 \%$. Outro resultado que merece destaque está na terceira dimensão do QASSaB, quando é analisada a satisfação dos usuários com o acesso ao serviço odontológico. Nesse item, a obtenção de uma consulta/vaga foi considerada fácil ou muito fácil pela maioria dos usuários da ESF (45\%); ao contrário, porém, a maioria dos usuários da UBS convencional (45\%) classificou o acesso como difícil ou muito difícil. O acesso apre- sentou diferença estatística quando a satisfação dos usuários com essa dimensão foi avaliada (valor de $p$ $0,03)$, demonstrando ser esse um importante componente para a satisfação dos usuários com o serviço de saúde bucal.

A limpeza dos ambientes físicos, tais como a sala de espera e os banheiros das unidades de saúde, também apresentou diferença estatisticamente significativa quando ESF e UBS convencional foram comparadas. Esse resultado demonstra a importância desse aspecto para a satisfação dos usuários com os serviços de saúde bucal. 
A sétima dimensão, referente à resolutividade do atendimento odontológico, também apresentou diferença quando foram confrontados os dados da ESF e da UBS convencional. Os usuários atendidos na UBS convencional, proporcionalmente, apresentaram maior satisfação com a sua capacidade mastigatória referida após o tratamento recebido, demonstrando que esse é, também, um importante marcador de satisfação com os serviços de saúde bucal.

As demais dimensões constantes no QASSaB não apresentaram diferença estatisticamente significativa, quando foi realizada uma análise comparativa entre o serviço de saúde bucal da ESF e o serviço de saúde bucal da UBS convencional.

A Tabela 4 apresenta, por fim, o escore total de satisfação dos usuários segundo a modalidade de serviço de saúde bucal por eles acessada. Considerando-se todas as dimensões do QASSaB, pode-se constatar que os escores apresentam-se elevados na modalidade ESF.

Tabela 4 - Escore total de satisfação dos usuários dos serviços odontológicos da ESF e UBS na cidade de Não-Me-Toque/RS, Brasil, 2011

\begin{tabular}{l|r|r|r|r|r}
\hline \multirow{2}{*}{$\begin{array}{l}\text { Satisfação em todas } \\
\text { as dimensões }\end{array}$} & \multicolumn{2}{|c|}{ ESF } & \multicolumn{2}{c|}{ UBS } & \multirow{2}{*}{$\begin{array}{l}\text { Valor } \\
\text { de p* }\end{array}$} \\
\cline { 2 - 5 } & $\mathrm{n}$ & \multicolumn{1}{c|}{$\%$} & $\mathrm{n}$ & \multicolumn{1}{c|}{$\%$} & 0,05 \\
\hline $\begin{array}{l}\text { Muito/ Totalmente } \\
\text { satisfeito }\end{array}$ & 109 & $93 \%$ & 140 & $86 \%$ & 0,05 \\
Apenas satisfeito & 8 & $7 \%$ & 23 & $14 \%$ & \\
Pouco satisfeito/ & 0 & $0 \%$ & 0 & $0 \%$ & \\
Insatisfeito & 117 & $100 \%$ & 163 & $100 \%$ & \\
Total & & & & \\
\hline
\end{tabular}

*Teste qui-quadrado com $5 \%$ de significância

\section{Discussão}

O processo de avaliar a satisfação do usuário é complexo e deve acontecer por meio de ferramentas apropriadas para esse fim. O instrumento designado a medir essa satisfação precisa ser baseado na compreensão do usuário quando expressa sua opinião sobre a natureza dos serviços que recebe. As expectativas dos usuários são dependentes do contexto do encontro clínico, da experiência passada e do conhecimento do paciente ${ }^{7}$.

Entende-se por satisfação a percepção e a avaliação que o usuário tem sobre dimensões definidas dos serviços de saúde. O grau de adesão ao tratamento ou às ações preventivas pode ser influenciado pelas relações interpessoais no cuidado, melhorando o desfecho terapêutico. Devido a esses fatos, torna-se importante incorporar a avaliação da satisfação do usuário como um dos componentes da qualidade do serviço ${ }^{8}$.

Além disso, grande parte da literatura disponível atualmente afirma que a ESF proporciona au- mento da satisfação dos usuários ${ }^{9-14}$. Entretanto, apesar da expressiva incorporação da Equipe de Saúde Bucal (ESB) na Saúde da Família, é necessário refletir se estão ocorrendo, de fato, mudanças no modelo assistencial em saúde bucal, caracterizado pelo atendimento individualizado, que visa apenas ao tratamento e que resiste à incorporação do conceito de cuidado em saúde.

Sem dúvida, uma das várias possibilidades de analisar as mudanças e a qualidade da atenção à saúde é pela avaliação do grau de satisfação dos usuários, pois ela é desenvolvida a partir de sua visão, que é a razão da existência de um serviço de saúde ${ }^{9}$. Isso posto, discutem-se as perspectivas encontradas neste estudo de base populacional.

Em relação à satisfação do usuário, foi detectada uma boa avaliação dos serviços de saúde bucal do SUS na cidade de Não-Me-Toque/RS. Entretanto, algumas diferenças entre as modalidades de serviço, que são a ESF e a UBS, foram observadas. Assim como no estudo de Gonçalves dos Santos ${ }^{5}$, realizado em 2006, na cidade de Curitiba/PR, foi constatado que, apesar da semelhança entre as UBSs com e sem ESF, elas proporcionaram níveis de satisfação diferentes em seus usuários.

No presente estudo, o enfoque foi a acessibilidade, pois, conforme Bamise et al. ${ }^{14}$ (2008), o fator que mais influencia a satisfação do usuário é o acesso aos serviços.

A acessibilidade foi uma dificuldade encontrada pelos usuários de Não-Me-Toque de uma forma geral. A ESF mostrou-se mais satisfatória no quesito obtenção de uma vaga/ficha para atendimento. $\mathrm{Na}$ ESF, a maioria dos usuários considerou fácil a obtenção de uma consulta; já na UBS, a situação inverte-se, pois a maioria classificou a obtenção de uma consulta como difícil.

Essa mesma característica foi observada no estudo de Oliveira et al..$^{9}$ (2009), realizado em Olinda/ $\mathrm{PE}$. Os autores objetivaram avaliar o grau de satisfação dos usuários quanto às ações de saúde bucal desenvolvidas nos serviços odontológicos das Unidades de Saúde da Família. Os resultados deste estudo revelam que, quanto à obtenção de uma ficha ou vaga para o atendimento no serviço, a maioria dos indivíduos acharam difícil ou muito difícil.

Conforme Moreira et al. ${ }^{15}$ (2005), a universalidade do acesso aos serviços de saúde bucal ainda parece uma realidade longínqua. A despeito dos avanços do SUS, principalmente com a implantação da ESF, o acesso à atenção odontológica necessita ser ampliado para grupos populacionais que têm como porta de entrada apenas os planos de saúde ou consultórios particulares, opções economicamente determinadas e socialmente excludentes ${ }^{15,16}$.

Os dados encontrados nesta pesquisa demonstram que, na maioria das dimensões estudadas, o resultado favorece a satisfação dos usuários da ESF, 
apesar de algumas não terem uma diferença estatisticamente significativa.

Em um escore médio das dimensões, percebe-se que todos os usuário sentem-se satisfeitos ou totalmente satisfeitos e nenhum indivíduo mostrou-se pouco satisfeito ou insatisfeito. Os usuários referem estar totalmente satisfeitos em $93 \%$ dos casos na ESF e em $86 \%$ dos casos na UBS. Isso sinaliza que, apesar de a satisfação atingir escores elevados, de uma forma geral, da população estudada, as pessoas que são cobertas pela ESF são mais satisfeitas do que as cobertas pela UBS tradicional.

O mesmo foi encontrado em Curitiba por Gonçalves dos Santos $^{5}$ (2006), em cujo estudo os usuários das Unidades de Saúde com ESF apresentaram uma satisfação maior com os serviços de saúde que os usuários das Unidades de Saúde sem ESF.

Destacam-se, portanto, os resultados referentes às relações humanas e ao acesso aos serviços de saúde bucal como variáveis importantes e associadas à satisfação dos usuários com os serviços de saúde.

\section{Conclusões}

De acordo com os resultados obtidos no presente estudo, pôde-se concluir que, em Não-Me-Toque/RS, os usuários cobertos pela ESF apresentam uma satisfação maior em algumas dimensões do QASSaB em comparação aos usuários cobertos pelas UBSs convencionais. Dentre os aspectos que se pode destacar, está a obtenção de vagas/fichas para o serviço, item no qual os usuários da ESF manifestaram-se mais satisfeitos que os da UBS.

Este estudo confirma o conceito de que o modelo de atenção reorganizador da Atenção Primária de Saúde implantado traz mais satisfação aos usuários, pois o profissional atua no território delimitado, realizando ações dirigidas aos problemas de saúde de maneira pactuada com a comunidade onde trabalha.

\section{Abstract}

Introduction: health evaluation is not regarded as routine in Brazil, however it is what provides grants and supports planning and decision-making of the National Health System. Objectives: to evaluate the access and satisfaction of users of the NHS oral health services in the city of Não-Me-Toque/RS, Brazil, comparing users of the Family Health Strategy (FHS) with the ones of the Basic Healthcare Centre (BHC). Methods: it was a crosssectional population based study with a sample of 280 individuals, identified in their homes through cluster sampling and users of dental public services. The Questionnaire for Quality Evaluation of Oral Health (QASSaB) - Questionário de Avaliação da Qualidade dos Serviços de Saúde Bucal, validated to Brazilian services was used. Results: chi-square tests showed that 93\% of users of the FHS were very/completely satisfied and only $7 \%$ were satisfied. In the group of users of the BHC, $86 \%$ were very/completely satisfied and the remaining $14 \%$ were satisfied. Conclusion: in Não-Me-Toque/RS, users with FHS coverage are more satisfied with oral health services than users covered by the $\mathrm{BHC}$.

Keywords: Patient satisfaction. Primary health care. Health evaluation. Health services.

\section{Referências}

1. Brasil. Ministério da Saúde. Secretaria de Atenção à Saúde. Departamento de Atenção Básica. Cadernos de Atenção Básica n. 17. Brasília: Ministério da Saúde; 2006.

2. Levcovitz E, Garrido NG. Saúde da família: a procura de um modelo anunciado. Cad Saúde Família 1996; 1:3-8.

3. Travesso-Yépez M, Morais NA. Reivindicando a subjetividade dos usuários da Rede Básica de Saúde: para humanização do atendimento. Cad Saúde Pública 2004; 20:80-8.

4. Brasil. Ministério da Saúde. Secretaria de Atenção Básica. Departamento de Atenção Básica. Avaliação na Atenção Básica em Saúde: caminhos da institucionalização. Brasília: Ministério da Saúde; 2005.

5. Gonçalves dos Santos ZFD. Avaliação da satisfação dos usuários dos serviços odontológicos do SUS, Curitiba, Paraná, Brasil [Tese de Doutorado]. Curitiba: Pontifícia Universidade Católica do Paraná; 2007.

6. Fernandes LMAG. Validação de um instrumento para avaliação da satisfação dos usuários, com os serviços públicos de saúde bucal - QASSaB [Tese de Doutorado]. Camaragibe/ PE: Universidade de Pernambuco; 2002.

7. Myburgh NG, Solanki GC, Smith MJ, Lalloo R. Patient satisfaction with health care providers in South Africa: the influences of race and socioeconomic status. Int J Qual Health Care 2005; 17:473-7.

8. Vieira-da-Silva LM, Formigli VLA. Avaliação em saúde: limites e perspectivas. Cad Saúde Pública 1994; 10:80-91.

9. Oliveira RS, Magalhães BG, Gaspar GS, Rocha RACP, Goés PSA. Avaliação do grau de satisfação dos usuários nos serviços de saúde bucal da Estratégia de Saúde da Família. Rev Bras Pesqui Saúde 2009; 11(4):34-8.

10. Baldani MH, Fadel CB, Possamai T, Queiroz MGS. A inclusão da Odontologia no Programa de Saúde da Família no Estado do Paraná, Brasil. Cad Saúde Pública 2005; 20:1026-35.

11. Elias PE, Ferreira CW, Alves MCG, Cohn A, Kishima V, Escrivão Jr. A, et al. Atenção Básica em Saúde: comparação entre PSF e ESB por estrato de exclusão social no município de São Paulo. Cien Saúde Colet 2006; 11:633-41.

12. Samico I, Hartz ZMA, Felisberto E, Carvalho EF. Atenção à saúde da criança: uma análise do grau de implantação e da satisfação de profissionais e usuários em dois municípios do estado de Pernambuco, Brasil. Rev Bras Saúde Mater Infant 2005; 5:229-40.

13. Trad LAB, Bastos ACS, Santana EM, Nunes MO. Estudo etnográfico da satisfação do usuário do Programa de Saúde da Família (PSF) na Bahia. Cien Saúde Colet 2002; 7:581-9.

14. Bamise CT, Bada TA, Bamise FO, Ogunbodede EO. Dental care utilization and satisfaction of residential university students. Libyan J Med 2008; 3(3):140-3. 
15. Moreira RS, Nico LS, Tomita NE, Ruiz T. A saúde bucal do idoso brasileiro: revisão sistemática sobre o quadro epidemiológico e acesso aos serviços de saúde bucal. Cad Saúde Pública 2005; 21:1665-75.

16. Stahlnacke K, Söderfeldt B, Unell L, Halling A, Axtelius B. Patient satisfaction with dental care in one Swedish age cohort, part II - what affects satisfaction. Swed Dent J 2007; 31(3):137-46.

Endereço para correspondência: Matheus Neves

Rua Dr. Salvador França, 667, complemento 304, Bairro Jardim Botânico

90690-00 Porto Alegre/RS

Fone: (51) 9625-0763

E-mail: matineves@gmail.com

Recebido: 02/01/2013. Aceito: 08/05/2013. 